\title{
Calibração de Redes Hidráulicas com Dados Transientes de Precisão Variável
}

\author{
John Kenedy de Araújo \\ UFC-CE-kenedy@ufc.br \\ Fazal Hussain Chaudhry \\ EESC-USP-fazal@sc.usp.br \\ Recebido: 19/11/02 - revisado: 06/05/03 - aceito: 04/06/03
}

\begin{abstract}
RESUMO
Este trabalho estuda a qualidade da estimação dos fatores de atrito em uma rede de distribuição de água existente a partir de dados observados de carga bidráulica. O Método Transiente Inverso (MTI) com um Algoritmo Genético emprega o Método das Características na solução das equações do movimento para escoamento transiente em redes de tubos. Para avaliar a confiabilidade do MTI desenvolvido aqui, uma rede exemplo simples estudada na literatura é usada para vários problemas de calibração relacionados à estimação dos fatores de atrito. A rede exemplo é composta de onze tubos, sete nós, e um reservatório. O comportamento transiente é imposto pela manobra de uma válvula em um dos nós. Vinte segundos de registros de cargas hidráulicas gerados pelo modelo transiente são consideradas como observacões sem erro no processo de calibração. Erros seguindo distribuição Gaussiana foram introduzidos nestes registros com diversos coeficientes de variação para representar defeitos nos instrumentos. A qualidade da estimação dos fatores de atrito é melhorada conforme o aumento do número de locais de medição. O desempenho do Algoritmo Genético é analisado utilizando as codificações binária e real para a representação dos parâmetros.
\end{abstract}

Palavras-chave: transientes em redes; método inverso; algoritmo genético.

\section{INTRODUÇÃO}

Modelos matemáticos são amplamente usados, na atualidade, por engenheiros e estudiosos de obras hidráulicas. No que concerne os sistemas de distribuição de água, a aplicação destes modelos engloba atividades de projeto, operação e manutenção em que a etapa de calibração refere-se à estimativa dos parâmetros para a modelação de um sistema existente.

Segundo Shamir e Howard (1968) a calibração de uma rede hidráulica de tubos consiste na determinação de características físicas e operacionais ideais de um sistema. Isto pode ser interpretado como a determinação de parâmetros tais que venham garantir a menor diferença entre cargas hidráulicas medidas e calculadas.

Desde a década de 1970, numerosas técnicas de calibração de parâmetros de modelos de redes tem sido propostos. Esses modelos de calibração podem ser classificados em três categorias: (1) procedimentos de tentativa e erro (com uso de equações analíticas); (2) modelos explícitos (simulação hidráulica); e (3) modelos implícitos (otimização). Vários autores reportam-se aos modelos da categoria (1): Walski, 1983 e Bhave, 1988 entre outros. O segundo grupo de modelos fundamenta-se na solução das equações da continuidade e da energia (Ormsbee e Wood, 1986a e 1986b; Boulos e Wood, 1990). O terceiro grupo de modelos de calibração é baseado em técnicas de otimização com a introdução de uma função objetivo.

A calibração das rugosidades em redes hidráulicas em uso pode ser feita usando métodos inversos baseados tanto em dados observados em regime permanente como transientes. Os transientes em redes hidráulicas ocorrem quando há variação da pressão e vazão provocada por um certo distúrbio, tal como, abertura e fechamento de uma válvula. Os transientes oferecem melhor oportunidade para realizar a calibração de redes por requererem menor número de locais de observação do que no caso de escoamento permanente, produzindo grande quantidade de informações no tempo. O Método Transiente Inverso (MTI) determina os fatores de atrito pela minimização dos desvios entre as cargas hidráulicas medidas e calculadas. Liggett e Chen (1994) aplicaram o MTI, em conjunto com o método de Levenberg-Marquardt para o ajuste entre as cargas medidas e calculadas. O método de Levenberg-Marquardt (Press et al. 1986) é uma técnica padrão que utiliza derivadas porém a sua solução depende do valor inicial atribuído e não garante a convergência ao mínimo global. Outros métodos de pesquisa para a otimização incluem os algoritmos estocásticos, tal como o Algoritmo Genético (AG). Goldberg (1989) e Michalewicz (1994) descrevem as carac- 
terísticas e principais empregos dos AG's na ciência e tecnologia.

Recentemente, Walter e Savic (1996) listaram as principais aplicações da otimização por AG em sistemas hidráulicos: projeto de redes de distribuição de água; regulação de pressão; calibração e sistemas de bombeamento. Reis et al. (1997) usaram o AG para regulação de pressão, com redução de vazamentos, através da posição ótima de válvulas de controle. Duas importantes vantagens do uso do AG são: a possibilidade de escolha entre um conjunto de soluções do espaço viável, aumentando significativamente as chances de encontrar o ótimo global e a não necessidade do uso de derivadas.

No processo de calibração é importante ressaltar que os dados observados devem ser de boa qualidade. $\mathrm{O}$ cenário ideal seria aquele onde não houvesse nenhum tipo de erro nas medidas. Na prática, a observação é cheia de dificuldades e erros oriundos de desajuste dos aparelhos, leituras erradas, etc.

A metodologia proposta neste trabalho usa o Método Transiente Inverso (MTI) para analisar a influência dos erros de medidas no processo de calibração dos fatores de atrito dos tubos de uma rede hidráulica. Um modelo conjugado hidráulico-otimização, baseado no Método das Características (MOC) e no Algoritmo Genético (AG), é aplicado. Analisa-se, também, a influência da aplicação do AG com representações binária e real.

\section{O MÉTODO TRANSIENTE INVERSO}

O escoamento transiente pode ser expresso matematicamente por duas equações: a equação da continuidade e a equação do movimento. Essas duas equações diferenciais permitem calcular os valores da vazão $Q$ e da carga hidráulica $H$ ao longo da tubulação $x$ e do tempo $t$. As equações podem ser resolvidas usando o Método das Características (MOC). Há muitas referências (Chaudhry, 1987; Wylie e Streeter, 1978; Karney e McInnis, 1992; Riggetto, 1994) que fornecem as deduções destas equações e ilustram o uso delas em simulações com fluxo em redes hidráulicas. As equações abaixo são equações diferenciais resultantes do processo de transformação pelo MOC e são válidas somente ao longo das linhas características:

$$
\begin{aligned}
& \frac{d H}{d t}+\frac{a}{g A} \frac{d Q}{d t}+\frac{f a}{2 g D A^{2}}|Q| Q=0 \\
& -\frac{d H}{d t}+\frac{a}{g A} \frac{d Q}{d t}+\frac{f a}{2 g D A^{2}}|Q| Q=0
\end{aligned}
$$

onde $H=$ carga hidráulica, $Q=$ vazão, $a=$ velocidade da onda ou celeridade, $g=$ aceleração da gravidade, $D=$ diâ- metro do tubo, $A=$ área da seção transversal do tubo, $f=$ coeficiente de atrito de Darcy-Weisbach, $t=$ tempo e $x=$ distância ao longo do tubo. Usando condições de contorno e as condições iniciais, todos os pontos da malha característica podem ser calculados usando uma forma integrada das equações 1 e 2.

No MTI desenvolvido por Liggett e Chen (1994), a informação transiente pode ser coletada em poucos locais da rede. Um determinado distúrbio no fluxo é transmitido à rede. As ondas transientes podem se deslocar com velocidades superiores a $1000 \mathrm{~m} / \mathrm{s}$. Essas ondas refletemse nas junções, válvulas e contornos do sistema. A coleta de informações pela propagação da onda transiente é a razão principal pela qual o MTI oferece maior potencial em comparação às técnicas de calibração em estado permanente.

Liggett e Chen (1994) desenvolveram a primeira aplicação do MTI usando técnicas com derivadas. Simpson et al. (2000) resolveram o mesmo problema usando um modelo combinado hidráulico-otimização (MOC e AG) na calibração dos fatores de atrito. Nash e Karney (1999) investigaram o MTI para tubos em série.

\section{ALGORITMO GENÉTICO}

\section{Generalidades}

Algoritmos Genéticos, AG’s, são métodos de otimização e busca inspirados nos mecanismos de evolução de populações de seres vivos. Foram introduzidos por John Holland (Holland, 1975) e difundidos por um dos seus alunos, David Goldberg (Goldberg, 1989). Estes algoritmos seguem o princípio de seleção natural e sobrevivência do mais apto, conforme Charles Darwin: "Quanto melhor um indivíduo se adaptar no seu meio ambiente, maior será sua chance de sobreviver e gerar descendentes" (“A Origem das Espécies”).

Uma das vantagens do uso de AG's é ser bastante versátil no que diz respeito à construção de novos operadores genéticos. Cada pesquisador pode obter seus próprios operadores tanto em codificação binária como real. O modelo de otimização por AG usado neste trabalho é descrito para os dois casos de codificação dos parâmetros e seus operadores genéticos.

O primeiro passo é gerar aleatoriamente uma população inicial que representa as possíveis soluções. A função objetivo $P$ do problema de otimização depende de $n$ parâmetros reais, cada um definido em um intervalo:

$$
\begin{aligned}
& P=P\left(x_{1}, x_{2}, \ldots, x_{n}\right) \\
& x_{i} \in\left[x_{i M I N}, x_{i M A X}\right] \text { e } i=1,2, \ldots, n
\end{aligned}
$$


onde $\chi_{i M I N}$ e $x_{i M A X}$ representam os valores mínimo e máximo de $x_{i}$, respectivamente.

O AG trabalha com uma estrutura de dados consistindo de uma população de indivíduos ou "cromossomos". Na literatura, há tradicionalmente duas maneiras de representar esta população: a representação binária e representação real. $\mathrm{Na}$ representação binária, o cromossomo é composto por uma cadeia de bits. A representação real é identificada geralmente por um vetor. Neste trabalho analisa-se o efeito das duas representações.

Dada uma população, a nova geração é produzida pelos seguintes operadores: seleção com elitismo, cruzamento, mutação e sucessão.

O procedimento de seleção com elitismo é o seguinte:

- $\quad$ Seja a população de $N C$ indivíduos $\left(a_{1}, a_{2}, \ldots\right.$, $a_{N C}$;

- A aptidão, $f\left(a_{i}\right)$, de cada indivíduo, é calculada conforme o valor da função objetivo;

- Faz-se o ordenamento decrescente da aptidão (para problema de maximização);

- Selecionam-se os melhores indivíduos deste ordenamento (Ex.: os 50\% primeiros) de maneira a formar uma população de $0,5 \times N C$ indivíduos. Estabelece-se, assim, uma probabilidade de seleção $p_{e}=0,5$;

- Forma-se o restante da população com uma escolha aleatória de indivíduos (dentre os melhores) que comporão a população de "pais".

Para a nova população selecionada, os operadores genéticos de cruzamento, mutação e sucessão são aplicados.

\section{- Representação binária}

O procedimento de cruzamento em codificação binária considerado aqui, conhecido como de um ponto, é aplicado a cada par de indivíduos (pais) com uma probabilidade $p_{c}$. Seja $a_{p}=\left(b_{1}, b_{2}, \ldots, b_{N C}\right)$ o primeiro pai e $a_{p}^{\prime}=\left(b_{1}^{\prime}, b_{2}^{\prime}, \ldots, b_{N C}^{\prime}\right)$ o segundo pai. Um número inteiro $J_{x}$ é gerado aleatoriamente entre 1 e $N B-1$ e os cromossomos dos pais (strings) são separados na posição $J_{x}$ :

$$
\begin{aligned}
& a_{p}=\left(b_{1}, b_{2}, \ldots, b_{J x} \mid b_{J x+1}, \ldots, b_{N B}\right) \\
& a_{p}^{\prime}=\left(b_{1}^{\prime}, b_{2}^{\prime}, \ldots, b_{J x}^{\prime} \mid b_{J x+1}^{\prime}, \ldots, b_{N B}^{\prime}\right)
\end{aligned}
$$

Assim produz-se, pela troca da segunda parte, os dois filhos $a_{f}$ e $a_{f}^{\prime}$ :

$$
\begin{aligned}
& a_{f}=\left(b_{1}, b_{2}, \ldots, b_{J x} \mid b_{J x+1}^{\prime}, \ldots, b_{N B}^{\prime}\right) \\
& a_{f}^{\prime}=\left(b_{1}^{\prime}, b_{2}^{\prime}, \ldots, b_{J x}^{\prime} \mid b_{J x+1}, \ldots, b_{N B}\right)
\end{aligned}
$$

A escolha do ponto de corte não é constante em toda a população, ou seja, para cada par de pais um novo número inteiro $J_{x}$ é gerado aleatoriamente.

O operador de mutação, conhecido como simples, é aplicado para cada bit individual com uma probabilidade $p_{m}$. Assim, se $b_{j y}$ é um bit selecionado para a mutação, então ele é substituído pelo seu complemento: $b_{j}{ }^{\prime}=1-b_{j}$.

\section{- Representação real}

O procedimento de cruzamento, conhecido como cruzamento aritmético (Michalewicz, 1994) é aplicado a cada par de indivíduos (pais) com uma probabilidade $p_{c}$. Seja o cromossomo $p_{1}$ o primeiro pai e $p_{2}$ o segundo pai. Os cromossomos filhos $c_{1}$ e $c_{2}$ são produzidos da seguinte forma:

$$
\begin{aligned}
& c_{1}=\beta p_{1}+(1-\beta) p_{2} \\
& c_{2}=(1-\beta) p_{1}+\beta p_{2}
\end{aligned}
$$

onde $\beta$ é um número gerado aleatória e uniformemente no intervalo $[0,1]$.

No processo seguinte, será considerada a mutação uniforme, ou seja, a simples substituição de um gene por um número aleatório. A mutação é aplicada para cada gene individual com uma probabilidade $p_{m}$. Dado um cromossomo $p$ com o $j$-ésimo gene selecionado para mutação, é produzido um cromossomo $c$ da seguinte forma:

$c_{1}=\left\{\begin{array}{lc}U\left(a_{i}, b_{i}\right), & \text { se } i=j \\ p_{\mathrm{i}} & \text { caso contrário }\end{array}\right.$

onde $a_{i}$ e $b_{i}$ representam os limites do intervalo permitido para o gene $c_{i}$ e $U\left(a_{i}, b_{i}\right)$ representa uma distribuição uniforme no intervalo $\left[a_{i}, b_{i}\right]$.

E, finalmente, como o último operador vem a sucessão. A população de indivíduos para a próxima geração é formada de $50 \%$ dos cromossomos dos pais e $50 \%$ dos cromossomos dos filhos, dentre aqueles de melhor aptidão. Estabelece-se, então, uma probabilidade de sucessão: $p_{s}=0,5$. 


\section{A Função Objetivo}

O AG necessita de uma função objetivo para o cálculo da aptidão de cada indivíduo. Cada indivíduo consiste de: bits (no caso de representação binária) e número real (representação real) que definem as características de uma solução particular. Neste trabalho, os fatores de atrito em cada tubo da rede são codificados nas duas representações. A aptidão de cada cromossomo representa o ajuste entre as cargas transientes medidas e calculadas através da soma das diferenças absolutas. Assim, a aptidão pode ser escrita como:

$$
f=-\sum_{j=1}^{N L} \sum_{i=1}^{N M}\left|H_{i, j}^{m}-H_{i, j}^{c}\right|
$$

onde $N M=$ número total de pontos; $N L=$ número de locais de medida na rede; $H_{i, j}{ }^{m}=$ carga medida; $H_{i, j}{ }^{c}=$ carga calculada; $i=1,2, \ldots, N M$; e $j=1,2, \ldots, N L$.

O objetivo é determinar a melhor combinação de fatores de atrito usando dados observados de forma precisa $(\sigma=0)$ e com erros de medida $(\sigma \neq 0)$, onde $\sigma$ é o desvio-padrão das medidas (imperfeições de leitura; defeito nos aparelhos, etc.).

\section{- Codificação dos parâmetros}

Para a codificação dos fatores de atrito é utilizado o esquema discreto estabelecido por Simpson et al. (2000). A escolha dos fatores de atrito está sujeita a um intervalo de valores discretos entre 0,010 e 0,040 com um incremento de 0,001 , totalizando 31 valores possíveis, conforme a Tabela 1.

Tabela 1 - Escolha do fator de atrito conforme o esquema discreto

\begin{tabular}{|c|c|c|c|c|c|}
\hline $\begin{array}{l}\text { N. }{ }^{\circ} \text { Intei- } \\
\text { ro }\end{array}$ & $f$ & $\begin{array}{l}\text { N. }{ }^{\circ} \text { Intei- } \\
\text { ro }\end{array}$ & $f$ & $\begin{array}{ll}\mathrm{N}^{\circ} & \text { Intei- } \\
\text { ro } & \\
\end{array}$ & $f$ \\
\hline 1 & 0,010 & 12 & 0,021 & 23 & 0,032 \\
\hline 2 & 0,011 & 13 & 0,022 & 24 & 0,033 \\
\hline 3 & 0,012 & 14 & 0,023 & 25 & 0,034 \\
\hline 4 & 0,013 & 15 & 0,024 & 26 & 0,035 \\
\hline 5 & 0,014 & 16 & 0,025 & 27 & 0,036 \\
\hline 6 & 0,015 & 17 & 0,026 & 28 & 0,037 \\
\hline 7 & 0,016 & 18 & 0,027 & 29 & 0,038 \\
\hline 8 & 0,017 & 19 & 0,028 & 30 & 0,039 \\
\hline 9 & 0,018 & 20 & 0,029 & 31 & 0,040 \\
\hline 10 & 0,019 & 21 & 0,030 & & \\
\hline 11 & 0,020 & 22 & 0,031 & & \\
\hline
\end{tabular}

Na codificação binária do presente estudo, o número mínimo de bits para representar um parâmetro com a precisão desejada é 5. Como cada cromossomo tem 11 variáveis, então o comprimento total do cromossomo é 55 .

\section{SEQÜÊNCIA PARA CALIBRAÇÃO VIA MTI}

A seqüência de passos da execução do modelo de calibração é a seguinte:

1. início;

2. geração aleatória de NC indivíduos;

3. codificação dos parâmetros;

4. cálculo das cargas hidráulicas transientes usando o MOC;

5. cálculo da aptidão de cada indivíduo;

6. determinação da nova geração através dos operadores de seleção com elitismo, cruzamento, mutação, e sucessão;

7. retorno ao passo (3) até que um número máximo de gerações ou um critério de convergência seja atendido;

8. fim.

\section{APLICAÇÃO DO MODELO}

Uma rede simples (Liggett e Chen, 1994) contendo onze tubos e sete nós (Figura 1) é usada para ilustrar o procedimento. A Tabela 2 apresenta as condições de estado permanente da rede. A última coluna descreve o tipo de nó. Demanda positiva indica fluxo de entrada na rede enquanto demanda negativa indica fluxo de saída. Os números entre parênteses e colchetes referem-se aos nós e tubos, respectivamente.

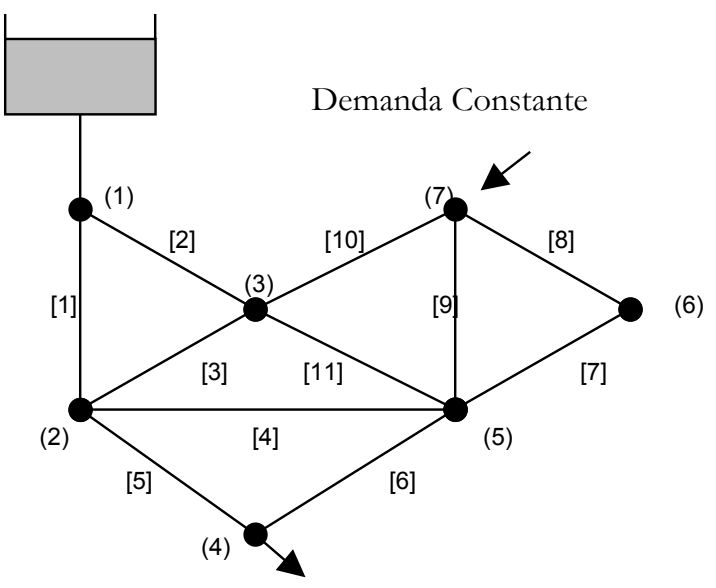

Vazão de saída (início do transiente)

Figura 1 - Rede hidráulica usada no exemplo 
A Tabela 3 apresenta as características físicas e as vazões iniciais. Os parâmetros dos tubos incluem o comprimento L, o diâmetro $\mathrm{D}$, as celeridades inicial e ajustada a e $a^{*}$, respectivamente, e os valores considerados corretos dos fatores de atrito.

Tabela 2 - Dados do estado permanente para os nós da rede.

\begin{tabular}{c|c|c|c}
\hline $\begin{array}{c}\text { Nó } \\
(1)\end{array}$ & $\begin{array}{c}H(\mathrm{~m}) \\
(2)\end{array}$ & $\begin{array}{c}\mathcal{Q}\left(\mathrm{m}^{3} / \mathrm{s}\right) \\
(3)\end{array}$ & $\begin{array}{c}\text { Descrição do Nó } \\
(4)\end{array}$ \\
\hline 1 & 30,00 & 0,046 & Reservatório de carga constante \\
2 & 29,73 & 0,000 & Junção comum \\
3 & 29,80 & 0,000 & Junção comum \\
4 & 29,43 & $-0,058$ & Válvula de controle \\
5 & 29,73 & 0,000 & Junção comum \\
6 & 29,74 & 0,000 & Junção comum \\
7 & 29,79 & 0,012 & Demanda constante \\
\hline
\end{tabular}

Tabela 3 - Características físicas dos tubos e vazões iniciais.

\begin{tabular}{c|c|c|c|c|c|c}
\hline $\begin{array}{c}\text { Tubo } \\
(1)\end{array}$ & $\begin{array}{c}Q_{\text {iniial }}\left(\mathrm{m}^{3} / \mathrm{s}\right) \\
(2)\end{array}$ & $\begin{array}{c}L(\mathrm{~m}) \\
(3)\end{array}$ & $\begin{array}{c}D(\mathrm{~m}) \\
(4)\end{array}$ & $\begin{array}{c}a(\mathrm{~m} / \mathrm{s}) \\
(5)\end{array}$ & $\begin{array}{c}a^{*}(\mathrm{~m} / \mathrm{s}) \\
(6)\end{array}$ & $\begin{array}{c}f \\
(7)\end{array}$ \\
\hline 1 & 0,023 & 305 & 0,250 & 1100,0 & 1560,5 & 0,0227 \\
2 & 0,023 & 215 & 0,250 & 1100,0 & 1100,0 & 0,0227 \\
3 & 0,008 & 215 & 0,200 & 1153,0 & 1153,0 & 0,0255 \\
4 & 0,001 & 305 & 0,200 & 1153,0 & 1560,5 & 0,0372 \\
5 & 0,029 & 215 & 0,250 & 1100,0 & 1100,0 & 0,0223 \\
6 & 0,029 & 215 & 0,250 & 1100,0 & 1100,0 & 0,0223 \\
7 & 0,003 & 215 & 0,200 & 1153,0 & 1153,0 & 0,0294 \\
8 & 0,003 & 215 & 0,150 & 1187,0 & 1187,0 & 0,0290 \\
9 & 0,011 & 305 & 0,250 & 1100,0 & 1560,0 & 0,0244 \\
10 & 0,001 & 215 & 0,150 & 1187,0 & 1187,0 & 0,0354 \\
11 & 0,014 & 215 & 0,250 & 1100,0 & 1100,0 & 0,0237 \\
\hline
\end{tabular}

O intervalo de tempo, $\Delta t$, para a simulação computacional foi de 0,2 s. O transiente foi simulado para um comprimento de registro de 20s. Com este intervalo de tempo, foram considerados 11 trechos (1 trecho para cada tubo) na rede. A coluna (5) da Tabela 3 apresenta os valores iniciais da celeridade nos tubos. Esses valores correspondem às seguintes características: espessura da parede do tubo, $e^{\prime}=10 \mathrm{~mm}$, coeficiente de Poisson, $v=0,25$, módulo de elasticidade do material do tubo, $E=120 \mathrm{GPa}$, módulo de elasticidade da água, $K=2,19 \mathrm{GPa}$, e massa específica da água, $\rho=999 \mathrm{~kg} / \mathrm{m}^{3}$. Seguindo a condição de estabilidade de Courant $(\Delta x>a \Delta t)$ e o método de ajuste da celeridade (Chaudhry, 1987), a coluna (6) apresenta os valores ajustados da celeridade. As condições iniciais (regime permanente) foram obtidas a partir de condições transientes usando o MOC.
O nó 4 contém uma válvula reguladora, cuja manobra provoca o transiente na rede. Seguindo Simpson et al. (2000), inicialmente a válvula está completamente aberta, com uma vazão de $58 \mathrm{l} / \mathrm{s}$, e mantida nesta posição até 2s. A partir daí, a válvula fecha-se linearmente em $8 \mathrm{~s}$ até atingir uma vazão de $27,5 \mathrm{l} / \mathrm{s}$. A válvula volta a abrir linearmente em 10s, atingindo a posição original.

A variação temporal da carga hidráulica, determinada a partir de modelagem hidráulica transiente via MOC, será considerada como observação "in loco" sem erros de medida. Os dados de carga sem erros de medida definidos desta forma são apresentados na Figura 2 para os nós 3, 5 e 6 .

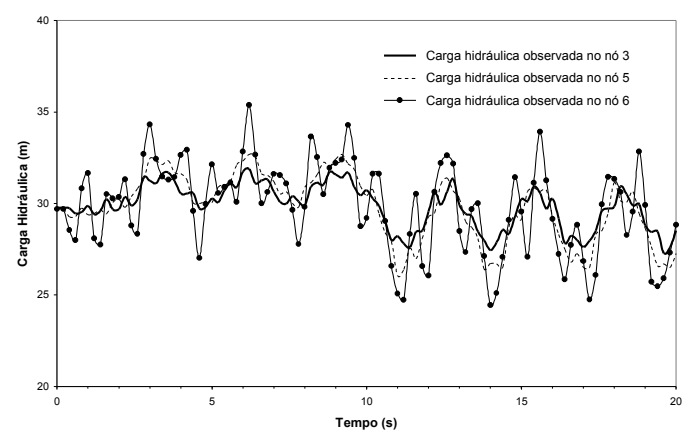

Figura 2 - Carga hidráulica “observada” para os nós 3, 5 e 6.

Para analisar a influência dos erros de medida nas cargas foram gerados ruídos de $0,5 \%, 1 \%, 5 \%$ e $10 \%$ na seqüência de dados sem erros. Esses ruídos foram obtidos de números aleatoriamente gerados por meio de uma distribuição normal padrão. Este procedimento tem o intuito de reproduzir os erros que ocorrem na prática.

\section{RESULTADOS}

Os parâmetros do AG aplicado para identificação de parâmetros foram: tamanho da população, $N C=100$; comprimento do string, $N B=55$; probabilidade de elitismo, $p_{e}=50 \%$; probabilidade de cruzamento, $p_{c}=100 \%$; probabilidade de mutação, $p_{m}=1 \%$; probabilidade de sucessão, $p_{s}=50 \%$; número de gerações, $N G=1000$. As estimativas dos fatores de atrito consistiram de uma média de 10 processamentos do AG usando diferentes conjuntos de números gerados a partir de sementes aleatórias diversas.

A calibração foi conduzida levando em consideração um local de observação (nó 2), três locais (nós 3, 5 e 6) e seis locais (nós 2, 3, 4, 5, 6 e 7) usando as duas representações para os parâmetros. A análise da influência dos erros nas medidas foi feita pelo Erro Médio Relativo (EMR) dado por 
$\operatorname{EMR}(\%)=\frac{1}{n}\left[\sum_{i=1}^{n} \frac{\left|f_{i}^{\text {correto }}-f_{i}^{\text {estimado }}\right|}{f_{i}^{\text {correto }}} \times 100\right]$

onde $i=$ número do tubo e $n=$ número total de tubos na rede.

O erro na função objetivo pode ser calculado da seguinte forma:

$E_{F O}(\%)=\left[\frac{\sum_{j=1}^{N L} \sum_{i=1}^{N M}\left|H_{i, j}^{m}-H_{i, j}^{c}\right|}{N L \times \bar{H}_{i, j}^{m}}\right] \times 100$

onde: $\bar{H}_{i, j}^{m}=$ carga média medida para 1; 3 e 6 locais.

As tabelas 4, 5 e 6 mostram os fatores de atrito estimados pela calibração via MTI, usando AG com representação real, com base nas informações sobre carga hidráulica observadas com e sem erros, respectivamente, no nó 2, nos nós 3, 5 e 6, e nos nós 2, 3, 4, 5, 6 e 7. Os resultados para representação binária podem ser vistos nas tabelas 7,8 e 9 .

Tabela 4 - Estimativas dos fatores de atrito obtidas do registro de carga hidráulica de 20s no nó 2 com vários graus de imprecisão nas observações usando o AG real.

\begin{tabular}{|c|c|c|c|c|c|c|}
\hline \multirow{4}{*}{$\begin{array}{l}\text { Tubo } \\
\text { (1) }\end{array}$} & \multirow{4}{*}{$\begin{array}{l}f_{\text {correto }} \\
\text { (2) }\end{array}$} & \multicolumn{5}{|l|}{ festimado } \\
\hline & & \multicolumn{5}{|c|}{ Erros de Observação } \\
\hline & & $0 \%$ & $0,5 \%$ & $1 \%$ & $5 \%$ & $10 \%$ \\
\hline & & $(3)$ & (4) & $(5)$ & $(6)$ & $(7)$ \\
\hline 1 & 0,0227 & 0,0243 & 0,0175 & 0,0165 & 0,0103 & 0,0100 \\
\hline 2 & 0,0227 & 0,0201 & 0,0287 & 0,0281 & 0,0208 & 0,0100 \\
\hline 3 & 0,0255 & 0,0283 & 0,0250 & 0,0238 & 0,0100 & 0,0100 \\
\hline 4 & 0,0372 & 0,0257 & 0,0103 & 0,0100 & 0,0101 & 0,0120 \\
\hline 5 & 0,0223 & 0,0236 & 0,0197 & 0,0183 & 0,0100 & 0,0100 \\
\hline 6 & 0,0223 & 0,0215 & 0,0242 & 0,0242 & 0,0221 & 0,0100 \\
\hline 7 & 0,0294 & 0,0299 & 0,0387 & 0,0382 & 0,0390 & 0,0390 \\
\hline 8 & 0,0290 & 0,0256 & 0,0383 & 0,0384 & 0,0390 & 0,0390 \\
\hline 9 & 0,0244 & 0,0232 & 0,0132 & 0,0102 & 0,0100 & 0,0100 \\
\hline 10 & 0,0354 & 0,0341 & 0,0109 & 0,0107 & 0,0100 & 0,0100 \\
\hline 11 & 0,0237 & 0,0255 & 0,0113 & 0,0111 & 0,0100 & 0,0100 \\
\hline $\operatorname{EMR}_{f}(\%)$ & - & 9,0 & 34,1 & 36,4 & 46,2 & 55,1 \\
\hline $\mathrm{E}_{\mathrm{FO}}(\%)$ & - & 0,7 & 38,0 & 76,2 & 380,8 & 756,6 \\
\hline
\end{tabular}

Tabela 5 - Estimativas dos fatores de atrito obtidas do registro de carga hidráulica de 20 s nos nós 3,5 e 6 com vários graus de imprecisão nas observações usando o AG real.

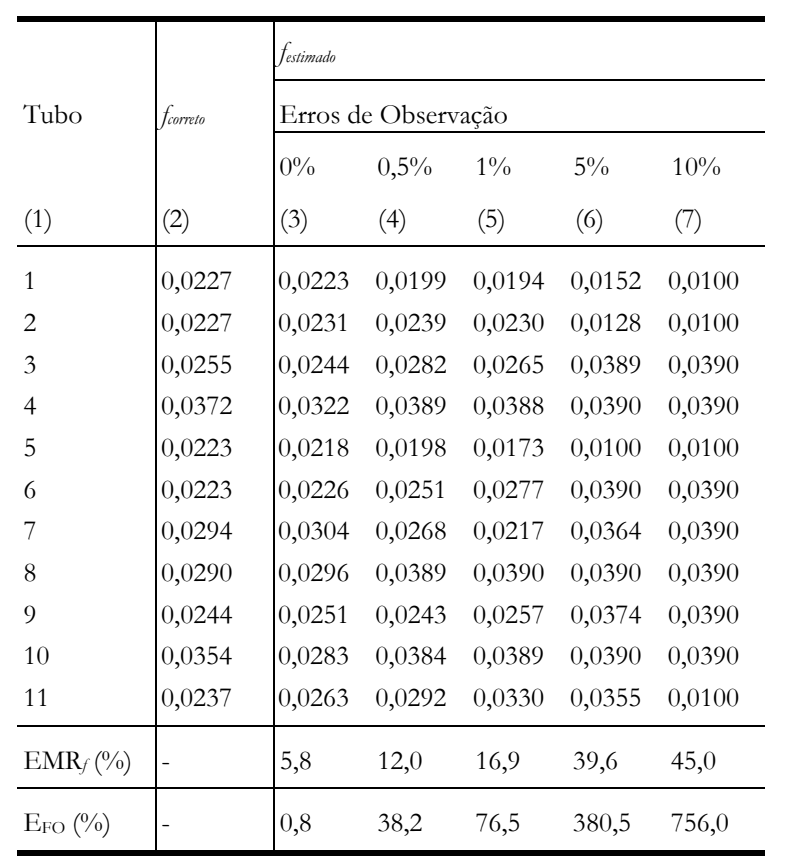

Tabela 6 - Estimativas dos fatores de atrito obtidas do registro de carga hidráulica de 20 s nos nós $2,3,4,5,6$ e 7 com vários graus de imprecisão nas observações usando o AG real.

\begin{tabular}{|c|c|c|c|c|c|c|}
\hline \multirow{3}{*}{$\begin{array}{l}\text { Tubo } \\
\text { (1) }\end{array}$} & \multirow{3}{*}{$\begin{array}{l}f_{\text {correto }} \\
(2)\end{array}$} & \multicolumn{5}{|l|}{$f_{\text {estimado }}$} \\
\hline & & \multicolumn{5}{|c|}{ Erros de Observação } \\
\hline & & $\begin{array}{l}0 \% \\
(3)\end{array}$ & $\begin{array}{l}0,5 \% \\
(4)\end{array}$ & $\begin{array}{l}1 \% \\
(5)\end{array}$ & $\begin{array}{l}5 \% \\
(6)\end{array}$ & $\begin{array}{l}10 \% \\
(7)\end{array}$ \\
\hline 1 & 0,0227 & 0,0229 & 0,0188 & 0,0191 & 0,0116 & 0,0100 \\
\hline 2 & 0,0227 & 0,0229 & 0,0241 & 0,0218 & 0,0104 & 0,0100 \\
\hline 3 & 0,0255 & 0,0254 & 0,0259 & 0,0205 & 0,0310 & 0,0100 \\
\hline 4 & 0,0372 & 0,0315 & 0,0384 & 0,0385 & 0,0388 & 0,0389 \\
\hline 5 & 0,0223 & 0,0222 & 0,0194 & 0,0172 & 0,0100 & 0,0100 \\
\hline 6 & 0,0223 & 0,0224 & 0,0242 & 0,0252 & 0,0271 & 0,0360 \\
\hline 7 & 0,0294 & 0,0288 & 0,0314 & 0,0342 & 0,0388 & 0,0390 \\
\hline 8 & 0,0290 & 0,0292 & 0,0330 & 0,0374 & 0,0390 & 0,0390 \\
\hline 9 & 0,0244 & 0,0251 & 0,0254 & 0,0255 & 0,0311 & 0,0390 \\
\hline 10 & 0,0354 & 0,0302 & 0,0294 & 0,0368 & 0,0384 & 0,0390 \\
\hline 11 & 0,0237 & 0,0229 & 0,0334 & 0,0363 & 0,0368 & 0,0100 \\
\hline $\operatorname{EMR}_{f}(\%)$ & - & 3,8 & 12,0 & 16,9 & 33,0 & 44,4 \\
\hline $\mathrm{E}_{\mathrm{FO}}(\%)$ & - & 0,7 & 38,4 & 76,8 & 381,5 & 757,9 \\
\hline
\end{tabular}


Tabela 7 - Estimativas dos fatores de atrito obtidas do registro de carga hidráulica de 20 s no nó 2 com vários graus de imprecisão nas observações usando o AG binário.

\begin{tabular}{|c|c|c|c|c|c|c|}
\hline \multirow{4}{*}{$\begin{array}{l}\text { Tubo } \\
\text { (1) }\end{array}$} & \multirow{4}{*}{$\begin{array}{l}f_{\text {correto }} \\
(2)\end{array}$} & \multicolumn{5}{|l|}{ festimado } \\
\hline & & \multicolumn{5}{|c|}{ Erros de Observação } \\
\hline & & $0 \%$ & $0,5 \%$ & $1 \%$ & $5 \%$ & $10 \%$ \\
\hline & & (3) & (4) & (5) & $(6)$ & $(7)$ \\
\hline 1 & 0,0227 & 0,0207 & 0,0158 & 0,0144 & 0,0100 & 0,0100 \\
\hline 2 & 0,0227 & 0,0256 & 0,0316 & 0,0316 & 0,0215 & 0,0100 \\
\hline 3 & 0,0255 & 0,0267 & 0,0218 & 0,0131 & 0,0100 & 0,0100 \\
\hline 4 & 0,0372 & 0,0253 & 0,0104 & 0,0100 & 0,0100 & 0,0109 \\
\hline 5 & 0,0223 & 0,0209 & 0,0183 & 0,0166 & 0,0100 & 0,0100 \\
\hline 6 & 0,0223 & 0,0231 & 0,0254 & 0,0266 & 0,0220 & 0,0100 \\
\hline 7 & 0,0294 & 0,0279 & 0,0396 & 0,0389 & 0,0400 & 0,0400 \\
\hline 8 & 0,0290 & 0,0330 & 0,0398 & 0,0397 & 0,0399 & 0,0400 \\
\hline 9 & 0,0244 & 0,0274 & 0,0139 & 0,0100 & 0,0100 & 0,0100 \\
\hline 10 & 0,0354 & 0,0247 & 0,0101 & 0,0114 & 0,0100 & 0,0100 \\
\hline 11 & 0,0237 & 0,0246 & 0,0119 & 0,0101 & 0,0100 & 0,0100 \\
\hline $\mathrm{EMR}_{f}(\%)$ & - & 12,1 & 38,6 & 45,1 & 46,7 & 56,0 \\
\hline $\mathrm{E}_{\mathrm{FO}}(\%)$ & - & 0,9 & 38,0 & 76,1 & 380,8 & 756,6 \\
\hline
\end{tabular}

Os EMR's dos valores estimados de fatores de atrito, usando representação real, em todas as tubulações (coluna 3 das tabelas 4, 5 e 6 ) apresentaram-se decrescentes nos três casos de disponibilidade de observações sem erros, quais sejam $9,0 \% ; 5,8 \%$; e 3,8\% na mesma ordem. No caso de representação binária, os EMR's (coluna 3 das tabelas 7,8 e 9) são iguais a $12,1 \% ; 5,2 \%$; e $5,6 \%$. O incremento de 3 para 6 locais de medida não melhora significativamente a estimação dos fatores. Os resultados para representação real demonstram claramente a importância da quantidade de informações para a estimação mais acertada dos parâmetros desconhecidos, no caso presente os fatores de atrito. Verifica-se, ainda, que o acréscimo do número de pontos de observações de 1 para 3 e de 3 para 6 resultou em redução do EMR da ordem de 35\% para representação real. No caso binário, a redução de 1 para 3 foi de 57,0\% enquanto de 3 para 6 não houve redução.

No caso de observações munidas de erros em termos de coeficientes de variação de diversas ordens $(0,5 \% ; 1 \% ; 5 \%$; e $10 \%)$, os erros de estimação, tanto na representação real como na binária, ampliam-se com o acréscimo dos erros de observações, independentemente do número de locais de observação. Especificamente, vê-se que os EMR's, no caso real, que eram 9,0\%; $5,8 \%$; e 3,8\% nos três casos de registros de observações sem erros passaram para $34,1 \% ; 12,0 \%$; e $12,0 \%$, respectivamente, com a presença de erros de apenas $0,5 \%$ do coeficiente de variação. $\mathrm{Na}$ representação binária, esta variação foi: 12,1\%;
5,2\%; 5,6\% (sem erros de observação) para 38,6\%; 13,4\%; $12,2 \%$ (com $0,5 \%$ de erros de observação). Desta forma, houve uma ampliação nos erros de estimação dos parâmetros de atrito em estudo, por um fator multiplicador de 3,8 ; 2,1; 3,1 (representação real) e 3,1; 2,6;2,2 (representação binária), devido ao erro de observação de $0,5 \%$. Fica mais evidente, na representação real, que a disponibilidade de observações em maior número de locais melhora, mesmo que de forma ínfima, a estimação dos parâmetros com a presença de erros nas cargas observadas.

Tabela 8 - Estimativas dos fatores de atrito obtidas do registro de carga hidráulica de 20 s nos nós 3,5 e 6 com vários graus de imprecisão nas observações usando o AG binário.

\begin{tabular}{|c|c|c|c|c|c|c|}
\hline \multirow{4}{*}{$\begin{array}{l}\text { Tubo } \\
\text { (1) }\end{array}$} & \multirow{4}{*}{$\begin{array}{l}f_{\text {correto }} \\
(2)\end{array}$} & \multicolumn{5}{|l|}{$f_{\text {estimado }}$} \\
\hline & & \multicolumn{5}{|c|}{ Erros de Observação } \\
\hline & & $0 \%$ & $0,5 \%$ & $1 \%$ & $5 \%$ & $10 \%$ \\
\hline & & (3) & (4) & (5) & (6) & $(7)$ \\
\hline 1 & 0,0227 & 0,0229 & 0,0211 & 0,0189 & 0,0157 & 0,0100 \\
\hline 2 & 0,0227 & 0,0226 & 0,0228 & 0,0236 & 0,0145 & 0,0100 \\
\hline 3 & 0,0255 & 0,0249 & 0,0196 & 0,0238 & 0,0400 & 0,0400 \\
\hline 4 & 0,0372 & 0,0348 & 0,0399 & 0,0398 & 0,0400 & 0,0400 \\
\hline 5 & 0,0223 & 0,0226 & 0,0198 & 0,0168 & 0,0100 & 0,0100 \\
\hline 6 & 0,0223 & 0,0226 & 0,0261 & 0,0282 & 0,0400 & 0,0400 \\
\hline 7 & 0,0294 & 0,0263 & 0,0298 & 0,0221 & 0,0382 & 0,0400 \\
\hline 8 & 0,0290 & 0,0292 & 0,0397 & 0,0400 & 0,0400 & 0,0400 \\
\hline 9 & 0,0244 & 0,0261 & 0,0237 & 0,0257 & 0,0394 & 0,0400 \\
\hline 10 & 0,0354 & 0,0263 & 0,0398 & 0,0399 & 0,0400 & 0,0400 \\
\hline 11 & 0,0237 & 0,0237 & 0,0302 & 0,0339 & 0,0258 & 0,0100 \\
\hline $\mathrm{EMR}_{f}(\%)$ & - & 5,2 & 13,4 & 19,0 & 37,9 & 47,2 \\
\hline $\mathrm{E}_{\mathrm{FO}}(\%)$ & - & 1,1 & 38,2 & 76,5 & 380,4 & 755,9 \\
\hline
\end{tabular}

No caso de observações munidas de erros em termos de coeficientes de variação de diversas ordens $(0,5 \% ; 1 \% ; 5 \%$; e $10 \%)$, os erros de estimação, tanto na representação real como na binária, ampliam-se com o acréscimo dos erros de observações, independentemente do número de locais de observação. Especificamente, vê-se que os EMR's, no caso real, que eram 9,0\%; 5,8\%; e 3,8\% nos três casos de registros de observações sem erros passaram para $34,1 \% ; 12,0 \%$; e $12,0 \%$, respectivamente, com a presença de erros de apenas $0,5 \%$ do coeficiente de variação. $\mathrm{Na}$ representação binária, esta variação foi: 12,1\%; $5,2 \% ; 5,6 \%$ (sem erros de observação) para 38,6\%; 13,4\%; $12,2 \%$ (com 0,5\% de erros de observação). Desta forma, houve uma ampliação nos erros de estimação dos parâmetros de atrito em estudo, por um fator multiplicador de 3,8; 2,1; 3,1 (representação real) e 3,1;2,6;2,2 (representação 
binária), devido ao erro de observação de $0,5 \%$. Fica mais evidente, na representação real, que a disponibilidade de observações em maior número de locais melhora, mesmo que de forma ínfima, a estimação dos parâmetros com a presença de erros nas cargas observadas.

Tabela 9 - Estimativas dos fatores de atrito obtidas do registro de carga hidráulica de 20 s nos nós $2,3,4,5,6$ e 7 com vários graus de imprecisão nas observações usando o AG binário.

\begin{tabular}{|c|c|c|c|c|c|c|}
\hline \multirow{4}{*}{$\begin{array}{l}\text { Tubo } \\
\text { (1) }\end{array}$} & \multirow{4}{*}{$\begin{array}{l}\text { correto } \\
(2)\end{array}$} & \multicolumn{5}{|l|}{ festimado } \\
\hline & & \multicolumn{5}{|c|}{ Erros de Observação } \\
\hline & & & & & & \\
\hline & & (3) & (4) & (5) & (6) & $(7)$ \\
\hline 1 & 0,0227 & 0,0220 & 0,0192 & 0,0190 & 0,0110 & 0,0100 \\
\hline 2 & 0,0227 & 0,0234 & 0,0235 & 0,0220 & 0,0107 & 0,0100 \\
\hline 3 & 0,0255 & 0,0245 & 0,0223 & 0,0188 & 0,0298 & 0,0100 \\
\hline 4 & 0,0372 & 0,0341 & 0,0396 & 0,0398 & 0,0395 & 0,0400 \\
\hline 5 & 0,0223 & 0,0221 & 0,0198 & 0,0170 & 0,0100 & 0,0100 \\
\hline 6 & 0,0223 & 0,0231 & 0,0247 & 0,0260 & 0,0277 & 0,0366 \\
\hline 7 & 0,0294 & 0,0303 & 0,0338 & 0,0374 & 0,0400 & 0,0400 \\
\hline 8 & 0,0290 & 0,0306 & 0,0334 & 0,0384 & 0,0400 & 0,0400 \\
\hline 9 & 0,0244 & 0,0237 & 0,0244 & 0,0234 & 0,0305 & 0,0400 \\
\hline 10 & 0,0354 & 0,0289 & 0,0335 & 0,0390 & 0,0398 & 0,0400 \\
\hline 11 & 0,0237 & 0,0257 & 0,0330 & 0,0371 & 0,0379 & 0,0100 \\
\hline $\mathrm{EMR}_{f}(\%)$ & - & 5,6 & 12,2 & 20,3 & 34,4 & 46,2 \\
\hline $\mathrm{E}_{\mathrm{FO}}(\%)$ & - & 1,2 & 38,4 & 76,7 & 381,5 & 757,9 \\
\hline
\end{tabular}

Para estabelecer o grau de influência na estimação de fatores de atrito nas tubulações da rede-exemplo, devido à eventual avariação de um determinado instrumento de medida, estudaram-se os erros médios relativos provocando erro de observação de $1 \%$ em nós individuais nos casos de registros de observações de carga obtidas em 3 e 6 locais de medida. As tabelas 10 e 11 apresentam as estimativas de fatores de atrito, caso de representação real, em todos os tubos e os EMR's devido às avariações individuais de instrumentos, enquanto que as tabelas 12 e 13 mostram estes resultados para a representação binária. No caso de representação real com 3 locais (Tabela 10), o EMR que era de $16,9 \%$ resultante de todos os instrumentos avariados, cai para $5,0 \% ; 5,1 \%$; e $4,8 \%$ quando se considera individualmente avariados os nós 3, 5 e 6 respectivamente. Não há uma variação muito grande nos valores, mas o nó 5 desponta como o nó mais sensível na propagação do erro de observação de carga para a estimação dos fatores de atrito, enquanto que o nó 6 contribui em grau menor na respectiva estimação. Na representação binária em 3 locais (Tabela 12), há uma equivalência entre os nós 3 e 5 en- quanto que o nó 6 propaga mais o erro. $\mathrm{Na}$ representação real com 6 locais (Tabela 11), o nó 7 é o mais sensível causando um EMR de 2,4\%. Na representação binária com 6 locais (Tabela 13), o nó 7, novamente, é o mais sensível com um EMR de 7,2\%. Nota-se que o nó 7 encontra-se em ponto mais distante em relação ao ponto de ocorrência do transiente. Em contrapartida, os nós menos sensíveis, situam-se próximos ao ponto de ocorrência do transiente. Este fato fica mais evidente na representação real. Assim, do ponto de vista de dimensionamento de um projeto de calibração de uma rede hidráulica via transiente, parece recomendável eleger como locais de observação, os nós próximos ao local de ocorrência de transientes para assegurar boa qualidade das estimativas de parâmetros da rede frente à ocorrência eventual de erros de observação.

Tabela 10 - Estimativas dos fatores de atrito obtidas do registro de carga hidráulica de 20 s nos nós 3,5 e 6 com imprecisão de $1 \%$ numa observação apenas e usando o AG real.

\begin{tabular}{|c|c|c|c|c|c|}
\hline \multirow[b]{2}{*}{$\begin{array}{l}\text { Tubo } \\
\text { (1) }\end{array}$} & \multirow[b]{2}{*}{$\begin{array}{l}f_{\text {forreto }} \\
\text { (2) }\end{array}$} & \multicolumn{4}{|l|}{$f_{\text {estitimado }}$} \\
\hline & & $\begin{array}{l}\text { Todos* } \\
\text { (3) }\end{array}$ & $\begin{array}{l}\text { Nó } 3 \\
(4) \\
\end{array}$ & $\begin{array}{c}\text { Nó } 5 \\
(5)\end{array}$ & $\begin{array}{c}\text { Nó } 6 \\
(6)\end{array}$ \\
\hline 1 & 0,0227 & 0,0194 & 0,0229 & 0,0226 & 0,0230 \\
\hline 2 & 0,0227 & 0,0230 & 0,0228 & 0,0228 & 0,0225 \\
\hline 3 & 0,0255 & 0,0265 & 0,0255 & 0,0240 & 0,0246 \\
\hline 4 & 0,0372 & 0,0388 & 0,0294 & 0,0285 & 0,0364 \\
\hline 5 & 0,0223 & 0,0173 & 0,0220 & 0,0221 & 0,0223 \\
\hline 6 & 0,0223 & 0,0277 & 0,0224 & 0,0227 & 0,0226 \\
\hline 7 & 0,0294 & 0,0217 & 0,0278 & 0,0270 & 0,0346 \\
\hline 8 & 0,0290 & 0,0390 & 0,0291 & 0,0293 & 0,0310 \\
\hline 9 & 0,0244 & 0,0257 & 0,0257 & 0,0256 & 0,0249 \\
\hline 10 & 0,0354 & 0,0389 & 0,0286 & 0,0334 & 0,0298 \\
\hline 11 & 0,0237 & 0,0330 & 0,0236 & 0,0245 & 0,0240 \\
\hline $\operatorname{EMR}_{f}(\%)$ & - & 16,9 & 5,0 & 5,1 & 4,8 \\
\hline $\mathrm{E}_{\mathrm{FO}}(\%)$ & $D^{-}$ & 76,5 & 26,4 & 26,4 & 26,0 \\
\hline
\end{tabular}

* imprecisões aplicados nos três nós simultaneamente

$\mathrm{Na}$ análise da performance das duas representações no algoritmo genético, os resultados mostram que a representação real produz menores valores de erros na estimação dos fatores de atrito, independentemente do número de locais de observação. Quanto ao erro na função objetivo $\left(E_{F O}\right)$, a representação real apresentou erros menores comparados àqueles obtidos pela representação binária nos casos de 1, 3 e 6 locais de observação sem a presença de erros nas cargas. Os valores dos erros na função objetivo, no caso da presença de desvios nas cargas, foram os mesmos nas duas representações. A Figura 3 apresenta o desempenho das duas representações em um 
dos 10 processamentos efetuados, utilizando-se somente o nó 2 como informação. $\mathrm{O}$ tempo médio de execução em micro-computador com processador Pentium com 128 MB de memória RAM, para este caso, usando representação real foi de 511,5 segundos enquanto que na representação binária este tempo foi de 519,7 segundos.

Tabela 11 - Estimativas dos fatores de atrito obtidas do registro de carga hidráulica de 20 s nos nós $2,3,4,5,6$ e 7 com imprecisão de $1 \%$ numa observação apenas e usando o AG real.

\begin{tabular}{|c|c|c|c|c|c|c|c|c|}
\hline \multirow{3}{*}{$\begin{array}{l}\text { Tubo } \\
\text { (1) }\end{array}$} & \multirow{3}{*}{$\begin{array}{c}f_{\text {correto }} \\
\text { (2) }\end{array}$} & \multicolumn{7}{|c|}{$f_{\text {estimado }}$} \\
\hline & & Todos* & Nó 2 & Nó 3 & Nó 4 & Nó 5 & Nó 6 & Nó 7 \\
\hline & & (3) & (4) & (5) & (6) & (7) & (8) & (9) \\
\hline 1 & 0,0227 & 0,0191 & 0,0230 & 0,0239 & 0,0228 & 0,0238 & 0,0230 & 0,0234 \\
\hline 2 & 0,0227 & 0,0218 & 0,0223 & 0,0218 & 0,0224 & 0,0218 & 0,0227 & 0,0222 \\
\hline 3 & 0,0255 & 0,0205 & 0,0248 & 0,0242 & 0,0273 & 0,0232 & 0,0230 & 0,0256 \\
\hline 4 & 0,0372 & 0,0385 & 0,0329 & 0,0340 & 0,0362 & 0,0315 & 0,0339 & 0,0302 \\
\hline 5 & 0,0223 & 0,0172 & 0,0224 & 0,0223 & 0,0227 & 0,0224 & 0,0218 & 0,0225 \\
\hline 6 & 0,0223 & 0,0252 & 0,0222 & 0,0219 & 0,0219 & 0,0222 & 0,0228 & 0,0220 \\
\hline 7 & 0,0294 & 0,0342 & 0,0283 & 0,0296 & 0,0287 & 0,0296 & 0,0291 & 0,0263 \\
\hline 8 & 0,0290 & 0,0374 & 0,0285 & 0,0284 & 0,0282 & 0,0289 & 0,0298 & 0,0282 \\
\hline 9 & 0,0244 & 0,0255 & 0,0252 & 0,0256 & 0,0249 & 0,0248 & 0,0248 & 0,0268 \\
\hline 10 & 0,0354 & 0,0368 & 0,0366 & 0,0302 & 0,0359 & 0,0347 & 0,0298 & 0,0309 \\
\hline 11 & 0,0237 & 0,0363 & 0,0240 & 0,0225 & 0,0231 & 0,0226 & 0,0246 & 0,0223 \\
\hline $\begin{array}{c}\mathrm{EMR}_{f} \\
(\%)\end{array}$ & - & 16,9 & 2,9 & 4,7 & 2,4 & 3,9 & 4,5 & 6,2 \\
\hline $\mathrm{E}_{\mathrm{FO}}(\%)$ & - & 76,8 & 13,5 & 13,7 & 13,4 & 13,6 & 13,4 & 13,6 \\
\hline
\end{tabular}

* imprecisões aplicados nos seis nós simultaneamente

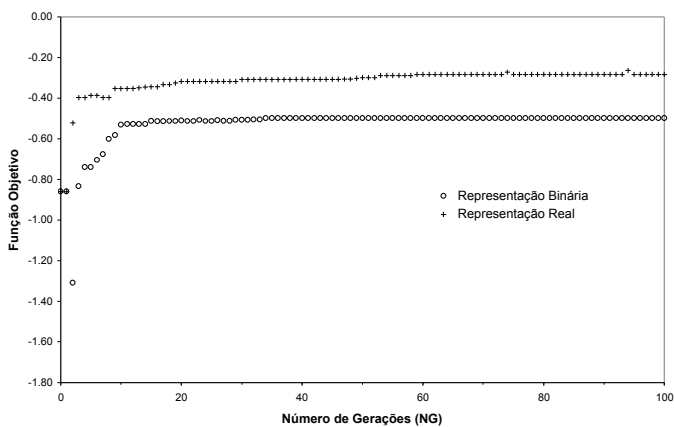

Figura 3 - Desempenho do AG real e binário
Tabela 12 - Estimativas dos fatores de atrito obtidas do registro de carga hidráulica de 20 s nos nós 3,5 e 6 com imprecisão de $1 \%$ numa observação apenas e usando o AG binário.

\begin{tabular}{|c|c|c|c|c|c|}
\hline \multirow{3}{*}{$\begin{array}{l}\text { Tubo } \\
\text { (1) }\end{array}$} & \multirow{3}{*}{$\begin{array}{l}\text { forreto } \\
(2)\end{array}$} & \multicolumn{4}{|l|}{$f_{\text {estimado }}$} \\
\hline & & Todos* & Nó 3 & Nó 5 & Nó 6 \\
\hline & & (3) & (4) & (5) & (6) \\
\hline 1 & 0,0227 & 0,0189 & 0,0218 & 0,0234 & 0,0218 \\
\hline 2 & 0,0227 & 0,0236 & 0,0236 & 0,0220 & 0,0240 \\
\hline 3 & 0,0255 & 0,0238 & 0,0290 & 0,0226 & 0,0275 \\
\hline 4 & 0,0372 & 0,0398 & 0,0367 & 0,0317 & 0,0353 \\
\hline 5 & 0,0223 & 0,0168 & 0,0227 & 0,0228 & 0,0212 \\
\hline 6 & 0,0223 & 0,0282 & 0,0223 & 0,0224 & 0,0235 \\
\hline 7 & 0,0294 & 0,0221 & 0,0280 & 0,0276 & 0,0299 \\
\hline 8 & 0,0290 & 0,0400 & 0,0287 & 0,0289 & 0,0309 \\
\hline 9 & 0,0244 & 0,0257 & 0,0252 & 0,0257 & 0,0268 \\
\hline 10 & 0,0354 & 0,0399 & 0,0279 & 0,0342 & 0,0190 \\
\hline 11 & 0,0237 & 0,0339 & 0,0234 & 0,0224 & 0,0264 \\
\hline $\operatorname{EMR}_{f}(\%)$ & - & 19,0 & 5,1 & 5,1 & 9,9 \\
\hline $\mathrm{E}_{\mathrm{FO}}(\%)$ & - & 76,5 & 26,7 & 26,5 & 26,0 \\
\hline
\end{tabular}

* imprecisões aplicados nos três nós simultaneamente

Tabela 13 - Estimativas dos fatores de atrito obtidas do registro de carga hidráulica de 20 s nos nós 2,3 , 4, 5, 6 e 7 com imprecisão de $1 \%$ numa observação apenas e usando o AG binário.

\begin{tabular}{|c|c|c|c|c|c|c|c|c|}
\hline \multirow[b]{2}{*}{$\begin{array}{l}\text { Tubo } \\
\text { (1) }\end{array}$} & \multirow[b]{2}{*}{$\begin{array}{l}f_{\text {forreto }} \\
(2)\end{array}$} & \multicolumn{7}{|l|}{$f_{\text {estimado }}$} \\
\hline & & $\begin{array}{l}\text { Todos* } \\
\text { (3) }\end{array}$ & $\begin{array}{l}\text { Nó } 2 \\
\text { (4) }\end{array}$ & $\begin{array}{l}\text { Nó } 3 \\
\text { (5) }\end{array}$ & $\begin{array}{c}\text { Nó } 4 \\
(6)\end{array}$ & $\begin{array}{c}\text { Nó } 5 \\
(7)\end{array}$ & $\begin{array}{l}\text { Nó } 6 \\
(8)\end{array}$ & $\begin{array}{c}\text { Nó } 7 \\
\text { (9) }\end{array}$ \\
\hline 1 & 0,0227 & 0,0190 & 0,0231 & 0,0231 & 0,0228 & 0,0207 & 0,0232 & 0,0221 \\
\hline 2 & 0,0227 & 0,0220 & 0,0224 & 0,0222 & 0,0224 & 0,0243 & 0,0219 & 0,0236 \\
\hline 3 & 0,0255 & 0,0188 & 0,0208 & 0,0226 & 0,0224 & 0,0282 & 0,0231 & 0,0243 \\
\hline 4 & 0,0372 & 0,0398 & 0,0339 & 0,0344 & 0,0364 & 0,0379 & 0,0330 & 0,0322 \\
\hline 5 & 0,0223 & 0,0170 & 0,0222 & 0,0219 & 0,0224 & 0,0221 & 0,0226 & 0,0217 \\
\hline 6 & 0,0223 & 0,0260 & 0,0230 & 0,0228 & 0,0229 & 0,0229 & 0,0226 & 0,0232 \\
\hline 7 & 0,0294 & 0,0374 & 0,0297 & 0,0288 & 0,0299 & 0,0295 & 0,0312 & 0,0284 \\
\hline 8 & 0,0290 & 0,0384 & 0,0298 & 0,0298 & 0,0299 & 0,0293 & 0,0295 & 0,0303 \\
\hline 9 & 0,0244 & 0,0234 & 0,0245 & 0,0249 & 0,0246 & 0,0246 & 0,0246 & 0,0251 \\
\hline 10 & 0,0354 & 0,0390 & 0,0312 & 0,0305 & 0,0284 & 0,0283 & 0,0319 & 0,0259 \\
\hline 11 & 0,0237 & 0,0371 & 0,0245 & 0,0273 & 0,0261 & 0,0277 & 0,0251 & 0,0260 \\
\hline $\mathrm{EMR}_{f}(\%)$ & - & 20,3 & 4,9 & 5,7 & 5,0 & 6,5 & 4,9 & 7,2 \\
\hline $\mathrm{E}_{\mathrm{FO}}(\%)$ & - & 76,7 & 14,1 & 14,0 & 13,9 & 13,9 & 13,8 & 13,9 \\
\hline
\end{tabular}

* imprecisões aplicados nos seis nós simultaneamente 


\section{CONCLUSÕES}

Este trabalho se propôs a estudar a qualidade da estimação dos fatores de atrito dos tubos de uma rede hidráulica usando dados transientes, de desvios variados, via Método Transiente Inverso com otimização por Algoritmo Genético. Estudou-se, ainda, as representações real e binária no desempenho do algoritmo genético.

Quanto mais amplo é o programa de observações de cargas na rede, menor o erro de estimação dos fatores de atrito, considerada a presença ou ausência de erros nas medidas. Este fato é mostrado mais claramente nos resultados obtidos com a representação real.

Alguns locais de observação da rede apresentam maior robustez em termos de propagação de erros de observação para os erros médios de estimação. Particularmente os nós próximos ao local de ocorrência do transiente fornecem registros de dados que conduzem às estimativas de maior qualidade dos parâmetros sob calibração. Com respeito à sensibilidade na propagação dos erros, os nós mais distantes do ponto de ocorrência do transiente indicam ser os mais sensíveis nesta propagação.

$\mathrm{Na}$ análise do desempenho do algoritmo genético com as representações real e binária, nota-se que a representação real apresenta melhores resultados tanto na estimativa dos fatores de atrito como nos valores da função objetivo. Além disso, a representação real consome menos tempo de processamento.

Finalmente, o processo de calibração via Método Transiente Inverso (MTI) utilizando-se algoritmos genéticos aponta como um procedimento promissor na avaliação do estado das redes de distribuição de água existentes que apresentam parâmetros hidráulicos modificados em razão da deteriorização de seus componentes.

\section{REFERÊNCIAS}

BHAVE, P. R. - Calibrating Water Distribution Network Models. Journal of Hidraulics Engineering, ASCE, v. 114, n. 1, p. 120-136, 1988.

BOUlOS, P. F., WOOD, D. J. - Explicit Calculation of PipeNetwork Parameters. Journal of Hidraulics Engineering, ASCE, v. 116, n. 11, p. 1329-1344, 1990.

CHAUDHRY, M. H. - Applied Hydraulic Transients. New York, Van Nostrand Reinhold Company, 1987. 521p.

GOLDBERG, D. E. - Genetic Algorithms in Search, Optimization and Machine Learning, 412 pp., Addison-Wesley, Reading, Mass., 1989.

HOLLAND, J. H. - Adaptation in Natural and Artificial Systems. MIT Press, 1975.

KARNEY, B. W., MCINNIS, D. - Efficient Calculation of Transient Flow in Simple Pipe Networks. Journal of Hidraulic Engineering, ASCE, v. 118, n. 7, p. 1014-1030, 1992.
LIGGETT, J. A., CHEN, L-C. - Inverse Transient Analysis in Pipe Networks. Journal of Hidraulic Engineering, ASCE, v. 120, n. 8, p. 934-955, 1994.

MICHALEWICZ, Z. - Genetic Algorithms + Data Structures = Evolution Programs, Second Edition, Springer-Verlag, Berlin, Germany, 1994.

NASH, G. A., KARNEY, B. W. - Efficient Inverse Transient Analysis in Series Pipe Systems. Journal of Hidraulic Engineering, ASCE, v. 125, n. 7, p. 761-764, 1999.

ORMSBEE, L. E., WOOD, D. J. - Explicit Pipe Network Calibration. Journal of the Water Resources Planning and Management, ASCE, v. 112, n. 2, p. 166-182, 1986a.

ORMSBEE, L. E., WOOD, D. J. - Implicit Pipe Network Calibration. Journal of Hidraulics Engineering, ASCE, v. 112, n. 12, p. 1195-1207, 1986b.

PRESS, W. H., B. P., FLANNERY, S. A. TEUKOLSKY, VETTERLING, W. T. - Numerical Recipes. Cambridge University Press, Cambridge, UK, 1986.

REIS, L. F. R., PORTO, R. M., CHAUDHRY, F. H. - Optimal Location of Control Valves in Pipe Networks by Genetic Algorithms. Journal of the Water Resources Planning and Management, ASCE, v. 123, n. 6, p. 317-326, 1997.

RIGHETTO, A. M. - Cálculo Hidráulico de Rede de Distribuição de Agua a partir de Condições Transitórias: Adequação e Estudo de Casos. São Carlos. Tese de Livre Docência - Escola de Engenharia de São Carlos, Universidade de São Paulo, 1994.

SHAMIR, U., HOWARD, C. D. D. - Water Distribution Systems Analysis. Journal of the Hydraulics Division, ASCE, v. 94, n. HY1, p. 219-234, 1968.

SIMPSON, A., VÍTKOVSKY, J., LAMBERT, M. - Transients for Calibration of Pipe Roughnesses Using Genetic Algoritms. $8^{\text {th }}$ International Conference on Pressure Surges: Safe Design and Operation of Industrial Pipe Systems. BHR Groups. Publications n. 39, p. 587-597, 2000.

WALSKI, T. M. - Technique for Calibrating Network Models. Journal of the Water Resources Planning and Management, ASCE, v. 109, n. 4, p. 360-372, 1983.

WALTERS, G. A., SAVIC, D. A. - Recent Applications of Genetic Algorithms to Water System Design, Hydraulic Engineering Software, W. R. Blain (Ed.), Comp. Mech. Publ., Southampton, v. VI, 1996.

WYLIE, E. B., STREETER, V. L. - Fluid Transients. New York, McGraw-Hill, 1978, 384p.

\section{Calibration of Hydraulic Pipe Systems using Transient Data with Variable Precision}

\section{ABSTRACT}

This paper studies the quality of estimation of friction factors in an existing water distribution network from transient bydraulic head observation. The Inverse Transient Method (ITM) based on a Genetic Algorithm employs the Method of Characteristics to solve 
RBRH - Revista Brasileira de Recursos Hídricos Volume 9 n.1 Jan/Mar 2004, 55-66

the equations of motion for transient flow in the network pipes. In order to evaluate the reliability of ITM developed here, a simple example network studied in literature is used for various calibration problems related to the estimation of friction factors. The example network is composed of eleven pipes, seven nodes, and one reservoir. The transient behavior is imposed by a valve maneuver at one of the nodes. 20-second records of hydraulic heads generated by the transient model are taken as observations without measurement errors for use in the calibration exercises. Observation errors following Gaussian distribution were introduced into these records for various coefficients of variation to represent measurements by defective instruments. It is found that the quality of estimation of friction factor is improved when transient head is observed at increasing number of locations. The behavior of the Genetic Algorithm is studied using binary and real implementations.

Keywords: transients in pipe-networks; inverse method; genetic algorithm. 